\title{
Levamisole Induced Autoimmunity
}

\author{
Nina Tello*, Jorge Alejandro Mateos-Parra and Alejandro Díaz-Borjón
}

Medicina Interna, Hospital Angeles Lomas, Edo. of Mexico

"Corresponding author: Nina Tello, Medicina Interna, Hospital Angeles Lomas, Edo. of Mexico, Tel: 525552469584; E-mail: nina.tello@gmail.com

Rec date: January 13, 2016; Acc date: January 26, 2016; Pub date: February 03, 2016

Copyright: @ 2016 Tello N, et al. This is an open-access article distributed under the terms of the Creative Commons Attribution License, which permits unrestricted use, distribution, and reproduction in any medium, provided the original author and source are credited.

\begin{abstract}
Background: We present the case of a patient with vitiligo that was treated with levamisol and presented with pure levamisole induced vasculopathy. The patient had no cocaine use and immunologic findings persisted for more than 4 years after the consumption of the drug.

Case Presentation: 70 year-old women with a history relevant for vitiligo. Shortly after initiating levamisole she developed extensive necrotic skin lesions, coagulopathy and thrombocytopenia. At the time she presented an hemorragic stroke that resulted in hemiparesia. Four months later, she presented with loss of consciousness and a mixed aphasia. A CT scan revealed the prior lesion only, an EEG showed abnormal activity. Her lab tests showed a positive lupus anticoagulant and p-ANCA. She was initially managed with phenytoin, levetiracetam and enoxaparin. She returned with a status epilepticus and was then managed with phenyotin, levetiracetam, and diazepam with no response. Considering a nonthrombotic manifestation of antiphospholipid syndrome methylprednisolone 1 gr per day for three doses were administered with improvement in language and motor function. She continued enoxaparin and prednisone with good results. Mycophenolate mofetil was later added to her treatment as a prednisone sparing agent. Lupus anticoagulant persists positive to this date.
\end{abstract}

Conclusions: Vasculopathy presenting as skin necrosis or purpura is a feature of both cocaine and levamisole use. There are very few cases of levamisole only induced vasculitis and even less in the adult population. It is conventionally believed that the sole therapeutic intervention needed is avoidance of levamisol exposure. In this case, the persistence of lupus anticoagulant for more than 4 years, the neurologic symptoms upon steroid suspension and immediate response to bolus led to the decision of maintenance immunosupresive therapy and anticoagulation. There is a subset of patients who may develop an autoimmune disease and should be observed and treated for longer periods.

Keywords: Levamisole drug-induced autoimmunity; Lupus anticoagulant; ANCA vasculopathy

\section{List of Abbreviations}

DEA: Drug Enforcement Agency; LCIV: Levamisole Contaminated Cocaine Induced Vasculitis; LIV: Levamisole Induced Vasculitis

\section{Background}

Levamisole is a synthetic imidazothiazole derivative with important immunomodulatory properties. It was used in the 1970s for treatment of rheumatoid arthritis and for the treatment of colon cancer. Levamisol induced vasculitis was first reported simultaneously in 1978 in two women treated for rheumatoid arthritis [1,2]. Subsequent reports in children treated for nephrotic syndrome, and adults treated for multiple autoimmune conditions including vitiligo, appeared shortly after [3-5]. It was removed from the US market in 2000 because of the common occurrence of agranulocytosis6. The US Drug Enforcement Agency (DEA) first detected levamisole in cocaine bricks in 2003, increasing to $73.2 \%$ in 20097 . The reasons for the introduction of levamisole in the cocaine market are multifactorial. During the following years more than 200 levamisole contaminated cocaine induced vasculitis (LCIV) cases were reported in literature [6-12]. The presence of levamisole is difficult to prove because it has a short halflife, only $5-7 \%$ can be detected in urine, and specific diagnostic tools (gas chromatography and mass spectrometry) are required [13]. Only 57 patients have levamisole confirmation [6-13].Treatment consists of stopping levamisole use and supportive measures. We aim to describe a new form of presentation of levamisle vasculopathy that persisted long after suspension.

\section{Case Report}

In 2009, a 70 year-old woman with a history of vitiligo in treatment with levamisole that four months before presentation was hospitalized for necrotic skin lesions, diagnosed and managed then as StevenJohnson syndrome attributed to levamisole treatment. At that time she also had a left temporo-parietal hemorrhagic stroke due to coagulopathy with thrombocytopenia that resulted in hemiparesia. Cutaneous lesions were surgically debrided, she additionally received parenteral steroids that were eventually tapered and suspended. She then presented to our institution with an altered mental status and recently acquired aphasia. Her physical exam was relevant for a transcortical mixed aphasia with predominance in motor component and a right hemiparesia. Her skin lesions were in a scarring phase and localized in nose, ears and legs. Her head CT-scan revealed an old left temporo-parietal hemorrhage. An electroencephalogram showed abnormal activity. Immunological lab tests were reported as follows: pANCA 1:160 with a negative result for antinuclear antibodies, mieloperoxidase and anti-proteinase 3, anti-DNA, Scl70, RNP, SSA, SSB. She also had a positive lupus anticoagulant with negative IgG, 
Page 2 of 3

IgA, IgM anticardiolipins, antiß2-glycoprotein and antiphosphatidilserine. She was initially managed with phenytoin, levetiracetam and enoxaparin. She responded adequately and was discharged. The patient had a urinary tract infection treated with ertapenem and presented again with a status epilepticus. She was then managed with phenyotin, levetiracetam, diazepam and ertapenem was suspended but there was no response. Considering a nonthrombotic manifestation of antiphospholipid syndrome methylprednisolone $1 \mathrm{gr}$ per day for three doses were administered with improvement in language and motor function and no further convulsive crisis. She continued enoxaparin and prednisone $60 \mathrm{mg}$ QD with good results. Mycophenolate mofetil $500 \mathrm{mg}$ BID was later added to her treatment as a prednisone sparing agent. Lupus anticoagulant persists positive up to this point.

\section{Discussion}

We present a patient with a positive history of levamisole treatment and no history of drug abuse. Clinical features in the skin are compatible with the original reports in children, and this is the fifth case in an adult. Lesions occurred in ears, nose and legs and evolved to a necrotic stage, biopsy was not taken due to the time elapsed between presentation and our evaluation. We believe the original diagnosis of Stevens-Johnson syndrome is unlikely since different mucosa were spared. Another hypercoagulable state is unlikely in this age group and viral hepatitis was ruled out. Reports of levamisole-induced manifestations included skin manifestations like retiform purpura with or without necrosis and/or hemorrhagic bullae, livedoid pattern, pyoderma gangrenosum, punched out ulcers with central eschars, lichenoid eruptions, arthralgias/arthritis, reversible posterior leukoencephalopathy, disseminated autoimmune disease with hepatosplenomegaly, and hemolytic anemia, agranulocytosis, and drug-induced ANCA-associated vasculitis. Levamisole induced vasculitis (LIV) has a very distinct presentation, with painful purpuric lesions over the extremities, trunk, back, cheeks, and preferentially involving the ear lobes [12]. Ear necrosis presented in 7 of the 11 cases of LIV [1,2,4-6,9-12,14]. Various neurological side effects have also been described during levamisole therapy, the most concerning of which is leukoencephalopathy, manifested as ataxia with decreased mental status. Lowering of the seizure threshold in murine models has been established [15]. The patients improved with discontinuation of treatment and steroids [8]. Immunological abnormalities are a constant feature. Laboratory features such as perinuclear antineutrophil cytoplasmic in high titers [16,17] and lupus anticoagulant $[5,14]$ appeared in our patient without positive specificities, although different antigen targets have been reported [17]. Rongoletti14 established the induction of such antibodies, as well as antiphospholipid antibodies in 4 patients who were negative before levamisol exposure. Skin biopsy specimens from purpuric plaques in these children revealed thrombotic vasculitis, leukocytoclastic vasculitis, and/or vascular occlusion [14]. Pathogenesis of the levamisole-induced vasculopathic purpura is unclear. It is reasonable to suspect that levamisole activates an immunological cascade with the loss of tolerance to self-antigen in a genetically predisposed individual. Levamisole stimulates antibodies formation increasing both $\mathrm{T} / \mathrm{B}$ cell and neutrophilic responses, which lead to increased chemotaxis, formation of autoantibodies and immune complex deposition that adhere to the blood vessels, degranulate and release toxic oxygen metabolites, eventually leading to vascular injury [12]. Levamisole induces serological autoimmune abnormalities, leading to secondary antiphospholipid syndrome and a coagulopathic state. As with other drug induced vasculitis /vasculopathy early withdrawal of the offending drug mostly leads to complete recovery while more advanced disease and late withdrawal of the drug may necessitate use of corticosteroid and/or immunosuppressive therapy [16]. Given the fact that patients develop a temporary antiphospholipid syndrome, antiagregation with aspirin could be a preventive measure to take. On the other hand, lesions heal spontaneously within 2 or 3 weeks after discontinuation of levamisole, although serological abnormalities persist up to 14 months. It is not known if these autoantibodies are pathogenic and can cause thrombosis, or if on the contrary, they represent only an epiphenomenon. We did not find any reported case of thrombotic events in this context [13]. Treatment has been documented in 52 of the 62 reviewed cases. Of these, $43 \%$ were treated with immunosuppressants (including steroids, cyclophosphamide, and azathioprine). Fifty-seven percent were given no immunomodulatory treatment, and the lesions resolved with discontinuation of the drug [18].

\section{Conclusion}

Vasculopathy presenting as skin necrosis or purpura is a feature of both cocaine and levamisole use. Doubt about the true etiologic substance in drug users has been elicited since only a few cases could confirm the presence of levamisol. There are very few cases of levamisol only induced vasculitis and even less in the adult population. In these cases it is conventionally believed that the sole therapeutic intervention needed is avoidance of levamisol exposure. However we found indirect evidence that vasculitis /vasculopathy may persist and be clinically manifested long after levamisole has been suspended. The persistence of lupus anticoagulant for more than 4 years, the neurologic symptoms upon steroid suspension and immediate response to bolus led to the decision of maintenance immunosupresive therapy and prophylactic anticoagulation. There is a subset of patients who may develop an autoimmune disease and should be observed and treated for longer periods.

\section{References}

1. Macfarlane DG, Bacon PA (1978) Levamisole-induced vasculitis due to circulating immune complexes. Br Med J 1: 407-408.

2. Scheinberg MA, Bezerra JB, Almeida FA, Silveira LA (1978) Cutaneous necrotising vasculitis induced by levamisole. Br Med J 1: 408.

3. Scheinfeld N, Rosenberg JD, Weinberg JM (2004) Levamisole in dermatology : a review. Am J Clin Dermatol 5: 97-104.

4. Menni S, Pistritto G, Gianotti R, Ghio L, Edefonti A (1997) Ear lobe bilateral necrosis by levamisole-induced occlusive vasculitis in a pediatric patient. Pediatr Dermatol 14: 477-479.

5. Laux-End R, Inaebnit D, Gerber HA, Bianchetti MG (1996) Vasculitis associated with levamisole and circulating autoantibodies. Arch Dis Child 75: 355-356.

6. Ullrich K, Koval R, Koval E, Bapoje S, Hirsh JM (2011) Five consecutive cases of a cutaneous vasculopathy in users of levamisole-adulterated cocaine. J Clin Rheumatol 17: 193-196.

7. Buchanan JA, Heard K, Burbach C, Wilson ML, Dart R (2011) Prevalence of levamisole in urine toxicology screens positive for cocaine in an innercity hospital. JAMA 305: 1657-1658.

8. Larocque A, Hoffman RS (2012) Levamisole in cocaine: unexpected news from an old acquaintance. Clin Toxicol (Phila) 50: 231-241.

9. Walsh NM, Green PJ, Burlingame RW, Pasternak S, Hanly JG (2010) Cocaine-related retiform purpura: evidence to incriminate the adulterant, levamisole. J Cutan Pathol 37: 1212-1219. 
10. Farhat EK, Muirhead TT, Chaffins ML, Douglass MC (2010) Levamisoleinduced cutaneous necrosis mimicking coagulopathy. Arch Dermato 146: $1320-1321$

11. Lee BL, Stone JH, Gimbel D, Khosroshahi A (2010) A 44-year-old woman with cutaneous bullae and extensive skin necrosis. Arthritis Care Res (Hoboken) 62: 1805-1811.

12. Khan TA, Cuchacovich R, Espinoza LR, Lata S, Patel NJ, et al. (2011) Vasculopathy, hematological, and immune abnormalities associated with levamisole-contaminated cocaine use. Semin Arthritis Rheum 41: 445-454

13. de la Hera I, Sanz V, Cullen D, Chico R, Petiti G, et al. (2011) Necrosis of ears after use of cocaine probably adulterated with levamisole. Dermatology 223: 25-28.

14. Rongioletti F, Ghio L, Ginevri F, Bleidl D, Rinaldi S, et al. (1999) Purpura of the ears: a distinctive vasculopathy with circulating autoantibodies complicating long-term treatment with levamisole in children. $\mathrm{Br} \mathrm{J}$ Dermatol 140: 948-951.

15. Rehni AK, Singh TG (2010) Levamisole-induced reduction in seizure threshold: a possible role of nicotinic acetylcholine receptor-mediated pathway. Naunyn Schmiedebergs Arch Pharmacol 382: 279-285.

16. Wiik A (2008) Drug-induced vasculitis. Curr Opin Rheumatol 20: 35-39.

17. Graf J, Lynch K, Yeh CL, Tarter L, Richman N, et al. (2011) Purpura, cutaneous necrosis, and antineutrophil cytoplasmic antibodies associated with levamisole-adulterated cocaine. Arthritis Rheum 63: 3998-4001.

18. Gulati S, Donato AA (2012) Lupus anticoagulant and ANCA associated thrombotic vasculopathy due to cocaine contaminated with levamisole: a case report and review of the literature. J Thromb Thrombolysis 34: 7-10. 